\title{
Clinical, Laboratory and Radiological Predictors of Unfavorable Hospital Admission Course for Diabetic Patients with COVID-19 Mohamed Sorour Mohamed ${ }^{* 1}$, Tamer M. Gouda ${ }^{1}$, Ali M. Hassanin ${ }^{2}$, Nahla Ibrahim Zidan ${ }^{3}$, Walaa Sabry Fouad ${ }^{4}$, Amr Shaaban Hanafy ${ }^{1}$, Hussein Mohammed Hussein ${ }^{1}$ \\ Departments of ${ }^{1}$ Internal Medicine, ${ }^{2}$ Radiodiagnosis, ${ }^{3}$ Clinical Pathology and ${ }^{4}$ Family Medicine, Faculty of Medicine, Zagazig University Hospital, Egypt. \\ *Corresponding author: Mohamed Sorour Mohamed, Mobile: +201123043446, Email: doctor037@yahoo.com
}

\begin{abstract}
Background: Patients with diabetes mellitus infected with the new coronavirus, severe acute respiratory syndrome coronavirus 2 (SARS-CoV-2), are at risk of high morbidity and mortality.

Objective: Our study aimed to address the clinical, laboratory and radiological predictors of hospital admission course for patients with diabetes mellitus with COVID19 infection at the time of admission.

Patients and Methods: This was a single center, retrospective study of patients with diabetes mellitus (DM) admitted with COVID-19 infection. Patients with unfavorable admission course were compared with those of favorable course regarding patient characteristics, clinical presentation, results of laboratory investigations and chest CT severity score. Univariate and multivariate analysis associated with the unfavorable course was performed.

Results: Among 141 patients with DM admitted with COVID19, 44 patients had unfavorable course. Those with the favorable course were significantly younger $(53.3 \pm 16.4)$ vs $(67.3 \pm 15.8)$ in the unfavorable course group, multivariate analysis revealed that age more than 65 , presence of hypertension, CT severity scoring, high HBA1c, AST, IL6, oxygen saturation less than $93 \%$ and low lymphocyte count were the independent predictors of the unfavorable hospital admission course.

Conclusions: Our finding suggests that old age, presence of hypertension, hypoxia at presentation, in addition to high HBA1c, AST, IL6, CT severity scoring and low lymphocyte count were significant predictors of unfavorable admission course in COVID19 patients with diabetes.
\end{abstract}

Keywords: Coronavirus, COVID19, Diabetes mellitus, Predictors, Prognosis.

\section{INTRODUCTION}

In December 2019, an outbreak of pneumonia in Wuhan city in China caused by a new coronavirus SARS-CoV-2 and the disease was named COVID-19 (coronavirus disease 2019). It has been spread quickly and pandemic was declared by WHO on March, $2020^{(1,2)}$. The clinical presentation of COVID19 range from being asymptomatic to severe lower respiratory tract disease, the more severe form of the disease is more common in elderly patients and those who have comorbidities like diabetes mellitus, hypertension and cardiovascular disease ${ }^{(3)}$.

Patients with diabetes mellitus are at risk of high morbidity and mortality from many infectious diseases, Moreover, diabetes mellitus has been associated with increased risk of death from pneumonia related complications ${ }^{(4,5)}$. Recent studies showed that diabetes mellitus increase mortality in patients with COVID19 ${ }^{(6)}$, and up to $20-30 \%$ of nonsurviving patients with COVID-19 had preexistent diabetes mellitus ${ }^{(3,7)}$.

To our best knowledge, very few publications can be found which address one or more of risk factors for mortality in patients with diabetes mellitus infected with COVID-19. However, it is still unknown the factors which associated with poor prognosis and mortality in the admitted diabetic patient. Our study aimed to study the clinical, laboratory and radiological predictors of hospital admission course for patients with diabetes mellitus infected with COVID19 at time of admission.

\section{PATIENTS AND METHODS}

In this retrospective study, we included all adult type 2 diabetic patients (141 patients) with laboratory-confirmed COVID-19 admitted to Zagazig University Hospital between 20 May 2020 and 20 July 2020.

A confirmed case was defined as a positive result on real time PCR assay of nasopharyngeal swab specimens for SARS-CoV-2. In our study, we aimed to identify the clinical, laboratory and radiological markers predicting the unfavorable course of the disease at the initial assessment of diabetic patients admitted to hospital for management of COVID19.

From medical records of admitted patients, we collected the data of:

- Patient characteristics at admission: age, gender, body mass index, job, smoking status, other comorbidities (hypertension, hyperlipidemia, asthma, cardiac or liver disease.....).

- Clinical presentation at admission: fever, cough, fatigue, dyspnea, diarrhea, sore throat and headache. 
- Measurements of vital signs (blood pressure, pulse, respiratory rate and temperature) and of oxygen saturation.

- Laboratory investigations: complete blood count (CBC) was identified by cell counter (Sysmex XN2000, Japan), liver function test, creatinine, urea, C-reactive protein (CRP), fasting blood sugar (FBS), HBA1c, 2 hours postprandial sugar (PPS), LDH were done on Cobas 6000 auto analyzer (Roche diagnostic, Germany), prothrombin time and fibrinogen were measured on blood coagulation analyzer, model CA1500 (Sysmex, Japan), interleukin 6 (IL-6) levels were analyzed by ELISA sandwich technique, using commercially available kit (Quantikine, R and D system, Inc. , Minneapolis, USA), D-dimer, sodium, potassium, arterial blood gases, ESR and real-time polymerase chain reaction (RT-PCR) for SARS-CoV-2.

\section{Molecular detection of SARS CoV-2 (RT-PCR):}

Nasopharyngeal swabs were collected for SARS-CoV-2 real time reverse transcription polymerase chain reaction. Extraction of viral RNA was performed by using QIAamp. DNA blood minikit, from QIAGEN (Switzerland). The extracted nucleic acid was reverse transcripted into cDNA, quantitative real time PCR analysis was performed using TaqMan real time PCR method. A house keeping gene (IPC) as an internal control was used for calibration. The amplification process was done by using COVID 19 primer design kit, from GENESIG, and performed on QIagen-Rotor gene Q real time PCR instrument, Germany. During the operation, the probes annealed to three target sequences: ORF1ab, nucleocapsid (N) and spike (S), two of the three genes must be positive to consider the sample as positive for COVID19.

- Initial chest CT scan finding done to all patients at admission to diagnose and evaluate the severity of the disease, CT severity score (CT-SS) was estimated.

\section{CT protocol:}

Chest CT imaging was performed on a 16detector CT scanner (Emotion; SIEMENS, Germany). The patients were examined in supine position. CT images were then acquired during a single inspiratory breath-hold. The scan started from the lung apex to the costophrenic angles. We used the following parameters: $1.0 \mathrm{~mm}$ section thickness for reconstruction, $1.0 \mathrm{~mm}$ gap, tube voltage $120 \mathrm{kV}$, tube current $350 \mathrm{mAs}$, FOV $36.8 \times 42.9 \mathrm{~cm}$.

\section{Chest CT image analysis:}

CT scans were reviewed in thin-sections to reach a decision. The predominant patterns on CT scans were categorized as ground glass opacification (GGO), crazy-paving pattern, consolidation, and linear opacities.
Other minor signs which are not typical for Covid-19 were also observed including bronchiectasia, cavitation, pleural effusion, pericardial effusion, pneumothorax and mediastinal lymphadenopathy. The pattern of the distribution of the pulmonary lesions was evaluated as peripheral (predominantly subpleural), central and diffuse. The number of lobes involved was determined also. To determine the severity of disease, we depended on the main findings of GGO, crazy-paving pattern and consolidation. Both lungs were divided into five zones (3 right lobes and two left lobes). This included right upper lobe, right middle lobe, right lower lobe, left upper lobe, and left lower lobe.

Every lung lobe was assigned a score according to the percentage of involvement as the following:

- Score 0: corresponding to $0 \%$ involvement.

- Score 1: less than 5\% involvement.

- Score 2: involvement of 5\% to less than $25 \%$.

- Score 3: involvement of $25 \%$ to less than $50 \%$.

- Score 4: involvement of $50 \%$ to less than $75 \%$.

- Score 5: involvement of $75 \%$ or more.

The summation of scores of the 5 zones resulted in a semi-quantitative evaluation for overall lung involvement (maximal CT score for both lungs was 25). For each patient the CT severity score was estimated ${ }^{(8)}$.

The unfavorable course include: death during hospital admission and the need for ICU admission due to multiorgan failure or the need for mechanical ventilation during their hospital admission.

\section{Ethical consent: An approval of the study was obtained from Zagazig University Academic and Ethical Committee.}

\section{Statistical Method}

All data were analyzed using SPSS version 20 software (SPSS Inc., Chicago, IL, USA). Continuous data were expressed as the mean \pm SD and median (range), and the categorical data were presented as a number (percentage). Continuous data were checked for normality by using Kolmogorov-Smirnov test. Continuous variables with or without normal distribution between favorable and unfavorable admission course were compared using student t-test and Mann-Whitney U test, respectively.

Categorized data of the two groups were compared using the Chi-square $\left(\chi^{2}\right)$ test. Odd ratio (OR) was used to estimate risk in univariate analysis with $95 \%$ confidence interval. If interval contain 1 , the $\mathrm{P}$ value of test statistics was non-significant. A Stepwise logistic regression analysis was performed to assess the influence of various risk factors on hospital admission course in multivariate model. Pvalue of less than 0.05 was considered as statistically 
significant. $\mathrm{P}<0.001$ was considered highly statistically significant and $\mathrm{P}>0.05$ was considered non-statistically significant.

\section{RESULTS}

A total of 141 patients with diabetes mellitus confirmed with COVID-19 were included in the study. In terms of the outcome, 17 patients died during hospital admission, 22 patients were mechanically ventilated during hospital admission, 5 patients were admitted to ICU due to multiorgan failure, the remaining 97 patients had favorable admission course. Those with the favorable course were significantly younger compared to the unfavorable course group. Those who experienced unfavorable hospital admission courses had significantly higher BMI and a higher prevalence of hypertension, hyperlipidemia and chronic pulmonary disease (Table 1).

Table (1): Baseline characteristics of the study population.

\begin{tabular}{|l|c|c|c|}
\hline & Favorable course (n=97) & Unfavorable course (n=44) & p- value \\
\hline $\begin{array}{l}\text { Age (years) } \\
\text { Mean } \pm \text { SD }\end{array}$ & $53.3 \pm 16.4$ & $67.3 \pm 15.8$ & $<0.001$ \\
\hline $\begin{array}{l}\text { Gender, N (\%) } \\
\text {-Male } \\
\text {-Female }\end{array}$ & $\begin{array}{l}51(52.57 \%) \\
46(47.42 \%)\end{array}$ & $\begin{array}{c}21(47.7 \%) \\
23(52.27 \%)\end{array}$ & $>0.05$ \\
\hline $\begin{array}{l}\text { BMI }(\mathrm{kg} / \mathrm{m} 2) \\
\text { Mean } \pm \text { SD }\end{array}$ & $29.6 \pm 2.45$ & $34.6 \pm 3.71$ & $<0.05$ \\
\hline $\begin{array}{l}\text { Smoking } \\
\mathrm{N}(\%)\end{array}$ & $21(21.65 \%)$ & $9(20.45 \%)$ & $>0.05$ \\
\hline $\begin{array}{l}\text { Hypertension } \\
\mathrm{N}(\%)\end{array}$ & $46(47.42 \%)$ & $32(72.72 \%)$ & $<0.005$ \\
\hline $\begin{array}{l}\text { Chronic lung disease } \\
\mathrm{N}(\%)\end{array}$ & $23(23.7 \%)$ & $16(36.36 \%)$ & $<0.05$ \\
\hline $\begin{array}{l}\text { Hyperlipidemia } \\
\mathrm{N}(\%)\end{array}$ & $38(39.17 \%)$ & $26(59.1 \%)$ & $<0.005$ \\
\hline
\end{tabular}

Fever and cough were the most common presenting symptoms of both groups, yet those with unfavorable course were more likely to present with shortness of breath, tachycardia and tachypnea at admission. Oxygen saturation at presentation was significantly lower in the unfavorable course group compared to favorable course. The baseline concentration of LDH, CRP, IL6, FBS, HBA1c, urea, creatinine, total WBCs count, neutrophil count, ALT, AST, fibrinogen and prothrombin time were significantly higher the in unfavorable course group, while lymphocyte and eosinophil counts were significantly lower compared to favorable course group. There were significant differences between the CT severity scoring between favorable course group and unfavorable course group. More than $90 \%$ of patients with unfavorable hospital admission course had an affection of four or five lobes at initial CT scan in contrast to only $46.3 \%$ of favorable course group who were presented by four or more lobe affection (Table 2). Figures $(1,2)$ showed CT scan and CT severity score for two patients with favorable and unfavorable course respectively. 
Table (2): Clinical laboratory and radiological parameters of the diabetic patients at admission.

\begin{tabular}{|c|c|c|c|}
\hline & Favorable course $(n=97)$ & Unfavorable course $(n=44)$ & p-value \\
\hline $\begin{array}{l}\text { Clinical presentation at admission } \mathrm{N}(\%) \\
\text { Fever } \\
\text { Cough } \\
\text { Dyspnea } \\
\text { Diarrhea } \\
\text { Headache } \\
\text { Fatigue } \\
\end{array}$ & $\begin{array}{c}76(78.35 \%) \\
50(51.54 \%) \\
46(47.42 \%) \\
8(8.24 \%) \\
19(19.58 \%) \\
66(68 \%)\end{array}$ & $\begin{array}{c}35(79.54 \%) \\
23(52.27 \%) \\
28(63.63 \%) \\
5(11.36 \%) \\
8(18.2 \%) \\
31(70.45 \%)\end{array}$ & $\begin{array}{l}>0.05 \\
>0.05 \\
<0.001 \\
>0.05 \\
>0.05 \\
>0.05\end{array}$ \\
\hline $\begin{array}{l}\text { Mean arterial blood pressure }(\mathrm{mmHg}) \\
\text { Heart rate }(\mathrm{bpm}) \\
\text { Respiratory rate }(\mathrm{rpm})\end{array}$ & $\begin{array}{c}93(86-107) \\
81(73-95) \\
19(16-22)\end{array}$ & $\begin{array}{l}95(85-107) \\
89(74-102) \\
22(18-29)\end{array}$ & $\begin{array}{l}>0.05 \\
<0.05 \\
<0.05\end{array}$ \\
\hline $\mathrm{O}_{2}$ saturation $\%$ & $0.95(0.94-0.97)$ & $0.90(0.86-0.95)$ & $<0.001$ \\
\hline IL6 $(\mathrm{pg} / \mathrm{mL})$ & $14.6(8.3-25.9)$ & $32.1(16.3-75.1)$ & $<0.001$ \\
\hline FBS (mg/dL) & $178(149-213)$ & $203(158-327)$ & $<0.001$ \\
\hline HBA1c \% & $7.8(6.5-10.3)$ & $9.3(6.9-12.7)$ & $<0.001$ \\
\hline CRP (mg/l) & $10.72(5.23,19.94)$ & $67.61(25.59-121.36)$ & $<0.001$ \\
\hline $\operatorname{ESR}(\mathrm{mm} / \mathrm{hr})$ & $47.0(22.2-67.6)$ & $53.0(33.3-78.3)$ & $>0.05$ \\
\hline $\mathrm{LDH}(\mathrm{U} / \mathrm{L})$ & $439(373.2-579.6)$ & $588(406.2-748.3)$ & 0.008 \\
\hline Creatinine (mg/dl) & $1.21(0.72-1.45)$ & $1.76(0.69-3.45)$ & 0.01 \\
\hline Urea $(\mathrm{mg} / \mathrm{dl})$ & $23.2(13.42-39.35)$ & $37.36(21.5,56.5)$ & 0.02 \\
\hline D-dimer (ug/mL) & $2.25(0.78,6.25)$ & $3.28(0.68,8.43)$ & $>0.05$ \\
\hline WBC count $\left(10^{9} / \mathrm{L}\right)$ & $4.92(3.95-7.23)$ & $7.14(4.21-9.92)$ & 0.01 \\
\hline Lymphocyte count $\left(10^{9} / \mathrm{L}\right)$ & $2.16(1.04-3.08)$ & $0.96(0.58-1.45)$ & $<0.001$ \\
\hline Neutrophils count $\left(10^{9} / \mathrm{L}\right)$ & $2.43(1.95-3.70)$ & $6.32(2.35-9.35)$ & $<0.05$ \\
\hline Eosinophil count $\left(\left(10^{9} / \mathrm{L}\right)\right.$ & $1.32(0.27-2.90)$ & $0.21(0-0.43)$ & $<0.05$ \\
\hline Hemoglobin $(\mathrm{g} / \mathrm{dl})$ & $11.9(11.2-15.0)$ & $12.3(11 .-16.1)$ & $>0.05$ \\
\hline Albumin (g/dL) & $3.82(3.25-4.27)$ & $3.96(3.33-4.29)$ & $>0.05$ \\
\hline ALT ( U/L) & $32.4(17.6-45.4)$ & $45.2(26.3-63.4)$ & $<0.05$ \\
\hline AST (U/L) & $36.5(28.4-59.3)$ & $62.3(34.2-91.2)$ & $<0.01$ \\
\hline Total bilirubin $(\mathrm{mg} / \mathrm{dL})$ & $0.92(0.72-1.23)$ & $1.11(0.83,1.42)$ & $>0.05$ \\
\hline Fibrinogen $(\mathrm{g} / \mathrm{L})$ & $3.7(3.2-4.28)$ & $4.55(3.25-5.15)$ & $<0.05$ \\
\hline Prothrombin time (seconds) & $12.4(11.4-12.9)$ & $13.2(11.6-14.8)$ & 0.009 \\
\hline Sodium $(\mathrm{mmol} / \mathrm{L})$ & $138.3(135.2-144.9)$ & 139.2(134.4- 143.7) & $>0.05$ \\
\hline Potassium (mmol/L) & $3.9(3.65-4.36)$ & $3.61(3.34 .4 .23)$ & $>0.05$ \\
\hline $\begin{array}{l}\text { CT severity score (CT-SS) } \\
\text { Mean } \pm \text { SD } \\
\text { Minimum } \\
\text { Maximum } \\
\end{array}$ & $\begin{array}{c}7.62 \pm 2.66 \\
3 \\
13\end{array}$ & $\begin{array}{c}14.43 \pm 2.69 \\
10 \\
19\end{array}$ & $<0.001$ \\
\hline $\begin{array}{l}\text { Number of lobes affection } \\
1 \text { lobe } \\
2 \text { lobes } \\
3 \text { lobes } \\
4 \text { lobes } \\
5 \text { lobes } \\
\end{array}$ & $\begin{array}{c}0 \\
3(3.09 \%) \\
37(38.14 \%) \\
36(37.11 \%) \\
9(9.27 \%)\end{array}$ & $\begin{array}{c}0 \\
0 \\
4(9.09 \%) \\
14(31.8 \%) \\
26(59.09 \%)\end{array}$ & $\begin{array}{l}<0.001 \\
<0.001 \\
>0.05 \\
<0.001\end{array}$ \\
\hline
\end{tabular}


(A)

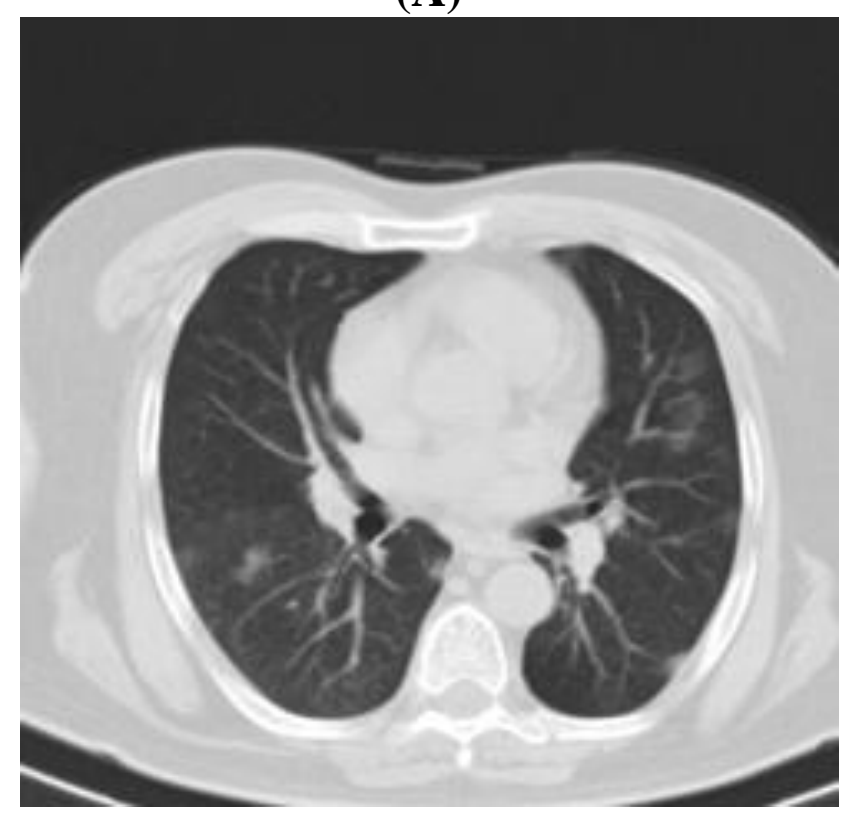

(C)

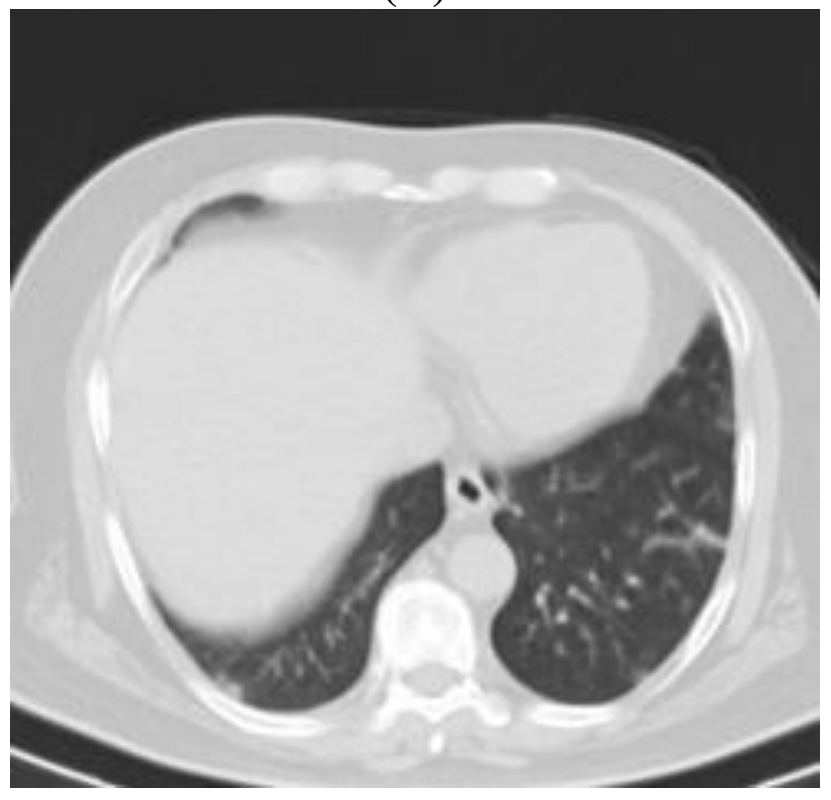

(B)

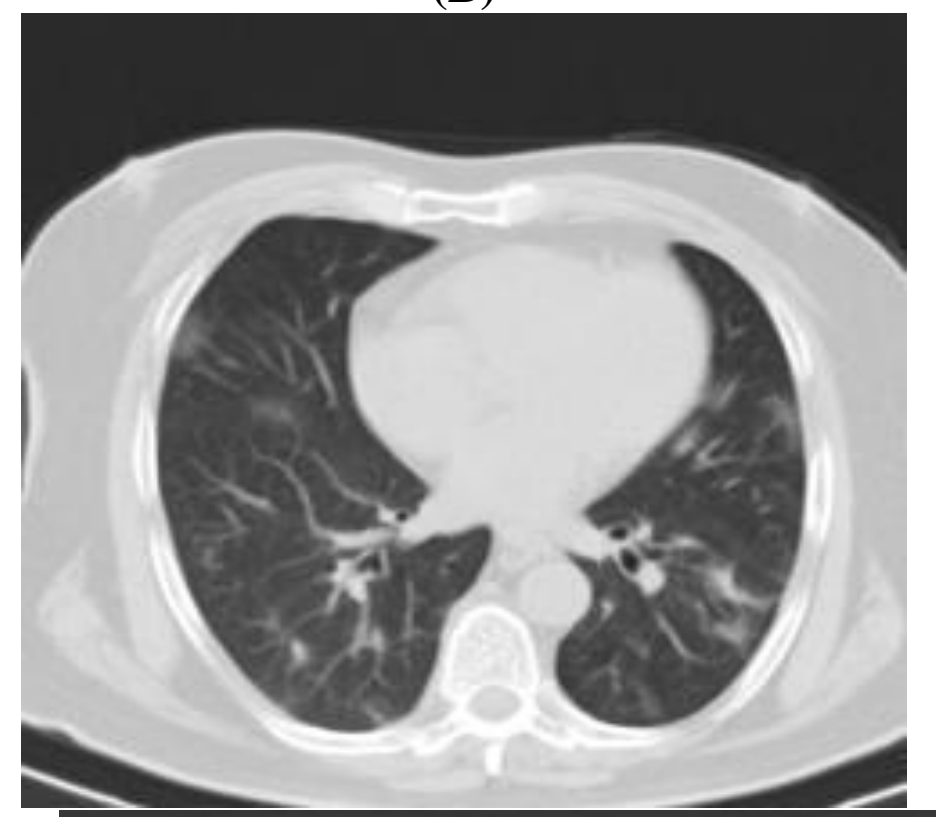

(D)

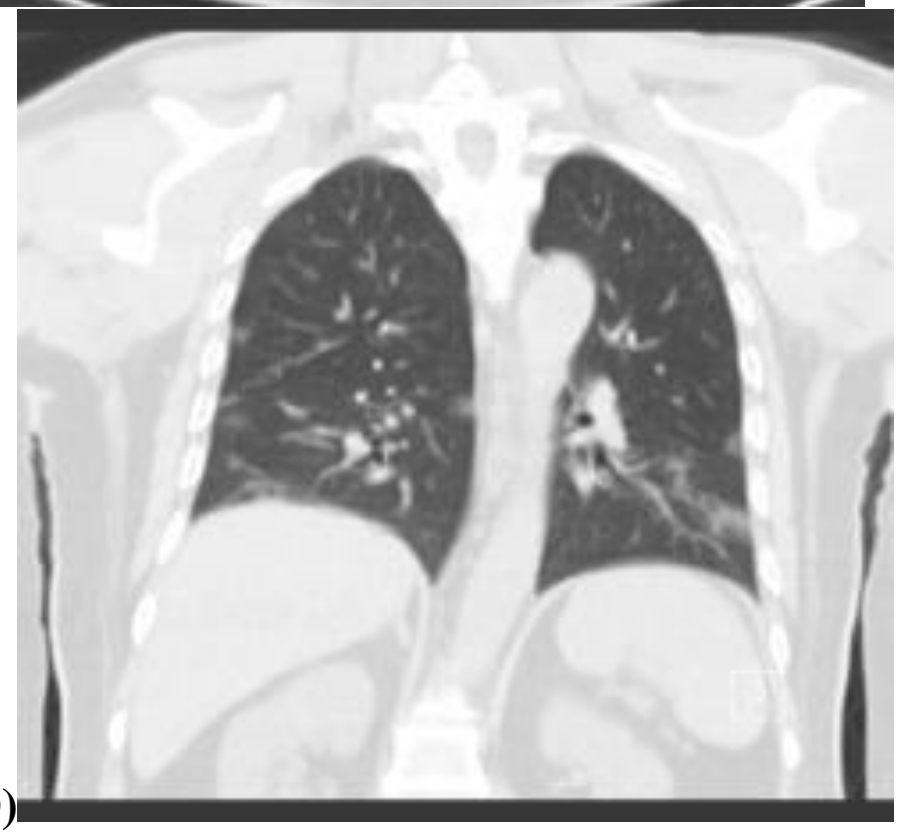

Figure (1): (A, B, C axial and D coronal): Male patient $50 \mathrm{y}$. Unenhanced chest CT scan shows bilateral and peripheral distribution of ground-glass opacities more dominant in the lower lobes. CT-SS was 7. The patient showed favorable course. 
(A)

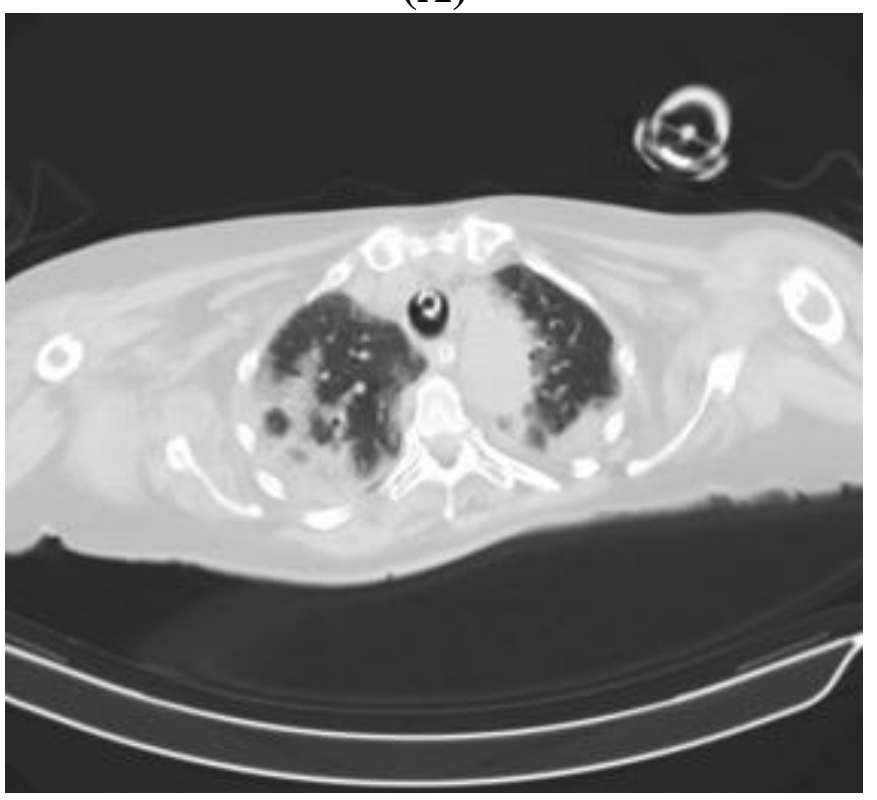

(C)

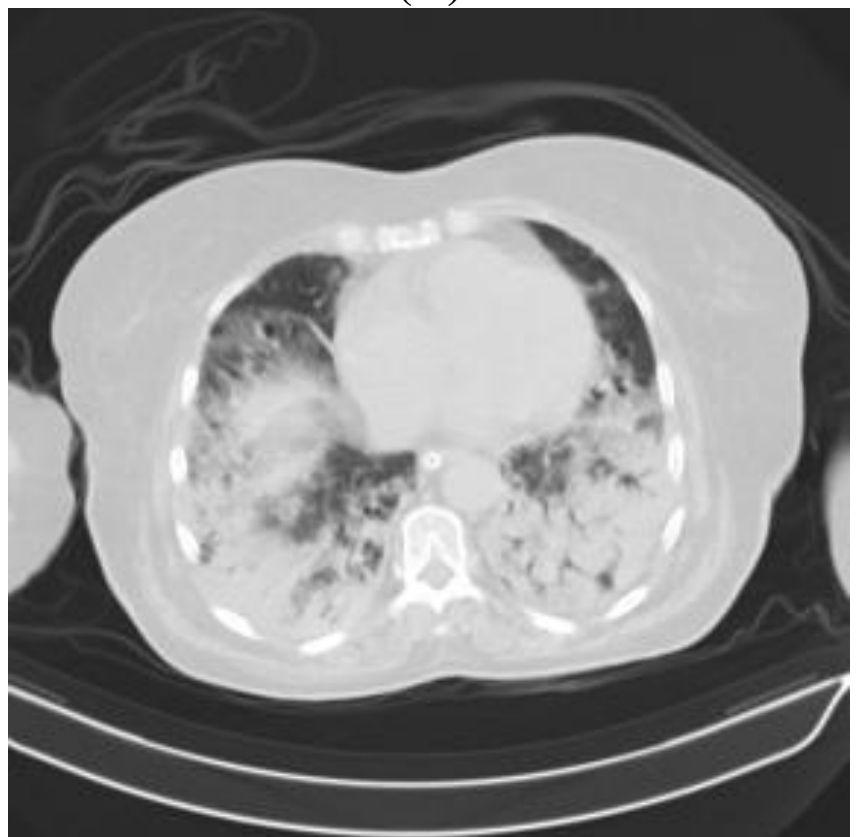

(B)

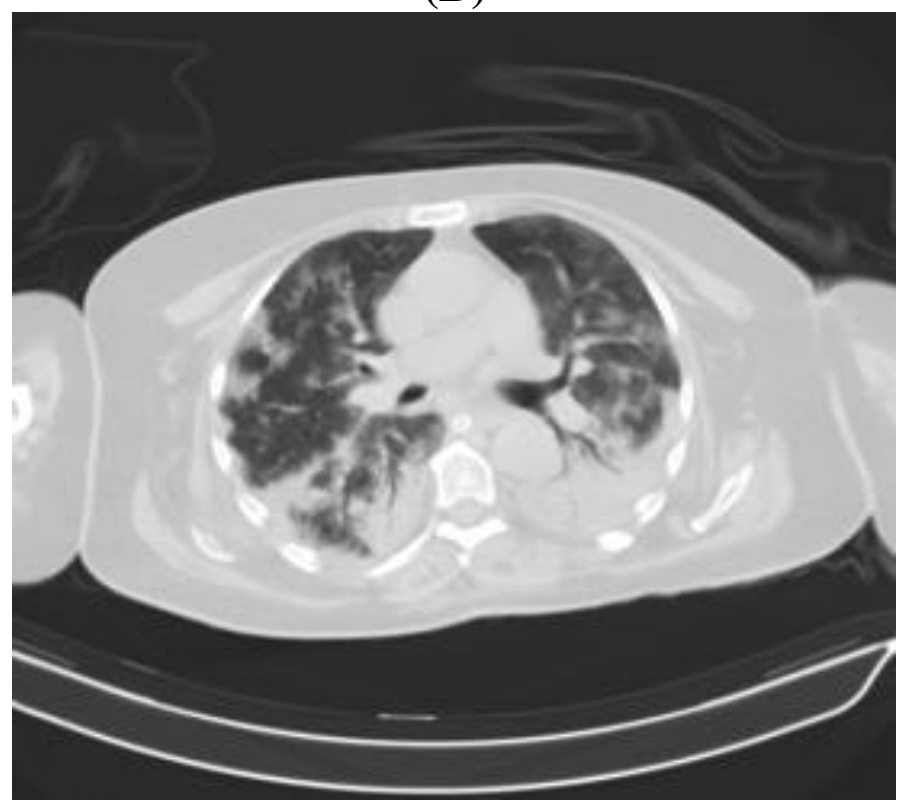

(D)

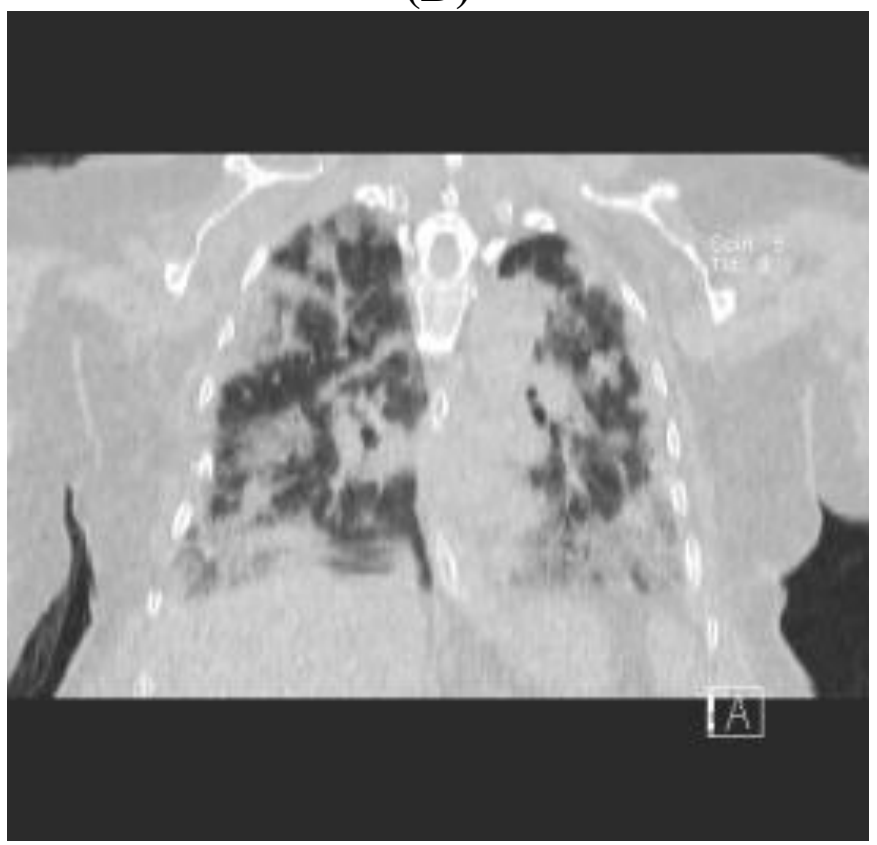

Figure (2): (A, B, C axial and D coronal): Female patient $62 \mathrm{y}$. Unenhanced chest CT scan shows bilateral and peripheral distribution of ground-glass opacities more dominant in the lower lobes and mounting to consolidation. Endotracheal tube is seen. CT-SS was 21. The patient showed unfavorable course and died.

Univariate analysis (Table 3) revealed significant increase in the risk of unfavorable admission course in patients older than 65, hypertensive, BMI more than 30, hypoxia with $\mathrm{SPO}_{2}$ less than $93 \%$, lymphopenia, higher HBA1C, WBCs count, IL6, ALT, AST, prothrombin time, creatinine and CT severity scoring. Based on stepwise multivariate logistic regression analysis (Table 4), age more than 65, presence of hypertension, high CT severity scoring, high HBA1c, AST, IL6, oxygen saturation less than $93 \%$ and low lymphocyte count were the independent predictors of unfavorable hospital admission course. 
Table (3): Risk estimation for possible risk factors for unfavorable outcome among diabetics.

\begin{tabular}{|l|c|c|l|c|c|}
\hline & OR & \multicolumn{3}{|c|}{ 95\% CI } & p \\
\hline Age $>$ 65 year & 2.91 & 1.62 & - & 4.72 & $<0.001$ \\
\hline Male sex & 1.19 & 0.78 & - & 1.24 & $>0.05$ \\
\hline BMI more than 30 & 1.72 & 1.21 & - & 2.37 & 0.009 \\
\hline Hypertension & 3.77 & 2.61 & - & 4.73 & $<0.001$ \\
\hline Chronic lung disease & 1.12 & 1.03 & - & 1.33 & $<0.05$ \\
\hline hyperlipidemia & 1.05 & 0.96 & - & 1.11 & $>0.05$ \\
\hline SPO ${ }_{2}$ less than 93\% & 0.93 & 0.91 & - & 0.96 & $<0.001$ \\
\hline HBA1c level & 2.41 & 1.9 & - & 3.62 & $<0.001$ \\
\hline WBC count $\left(10^{9} / \mathrm{L}\right)$ & 1.34 & 1.1 & - & 1.78 & 0.01 \\
\hline Lymphocyte count $\left(10^{9} / \mathrm{L}\right)$ & 0.47 & 0.12 & - & 0.79 & $<0.001$ \\
\hline Prothrombin time (seconds) & 1.21 & 1.01 & - & 1.31 & $<0.05$ \\
\hline IL6 (pg/mL) & 1.68 & 1.43 & - & 2.12 & $<0.001$ \\
\hline CRP (mg/l) & 1.03 & 0.89 & - & 1.34 & $>0.05$ \\
\hline LDH (U/L) & 1.09 & 0.78 & - & 1.37 & $>0.05$ \\
\hline ALT ( U/L) & 1.29 & 1.12 & - & 1.63 & 0.02 \\
\hline AST ( U/L) & 1.43 & 1.32 & - & 1.71 & $<0.001$ \\
\hline Creatinine (mg/dl) & 1.12 & 1.01 & - & 1.22 & $<0.05$ \\
\hline eosinophil count (10/L) & 1.02 & 0.89 & - & 1.19 & $>0.05$ \\
\hline CT severity score & 1.81 & 1.12 & - & 3.04 & $<0.001$ \\
\hline
\end{tabular}

Table (4): Multivariate logistic regression analysis for possible risk factors as predictors of unfavorable outcome among diabetics.

\begin{tabular}{|l|c|c|l|c|c|}
\hline & OR & \multicolumn{3}{|c|}{ 95\% CI } & p \\
\hline Age $>$ 65 year & 1.92 & 1.32 & - & 3.22 & $<0.05$ \\
\hline BMI more than30 & 1.17 & 0.98 & - & 1.27 & $>0.05$ \\
\hline Hypertension & 1.98 & 1.62 & - & 3.11 & $<0.001$ \\
\hline Chronic lung disease & 1.03 & 0.98 & - & 1.14 & $>0.05$ \\
\hline SPO2less than 93\% & 0.95 & 0.93 & - & 0.98 & $<0.001$ \\
\hline HBA1c level & 2.21 & 1.86 & - & 3.71 & $<0.001$ \\
\hline WBC count $\left(10^{9} / \mathrm{L}\right)$ & 1.18 & 0.95 & - & 1.33 & $>0.05$ \\
\hline Lymphocyte count $\left(10^{9} / \mathrm{L}\right)$ & 0.63 & 0.24 & - & 0.87 & 0.01 \\
\hline Prothrombin time $($ seconds) & 1.09 & 0.96 & - & 1.13 & $>0.05$ \\
\hline IL6 (pg/mL) & 1.82 & 1.53 & - & 2.47 & $<0.001$ \\
\hline CRP $(\mathrm{mg} / \mathrm{l})$ & 0.98 & 0.87 & - & 1.16 & $>0.05$ \\
\hline LDH $(\mathrm{U} / \mathrm{L})$ & 1.05 & 0.82 & - & 1.19 & $>0.05$ \\
\hline ALT $(\mathrm{U} / \mathrm{L})$ & 1.03 & 0.91 & - & 1.15 & $>0.05$ \\
\hline AST $(\mathrm{U} / \mathrm{L})$ & 1.13 & 1.02 & - & 1.29 & $<0.001$ \\
\hline Creatinine $(\mathrm{mg} / \mathrm{dl})$ & 1.02 & 0.96 & - & 1.09 & $>0.05$ \\
\hline CT severity score & 1.75 & 1.23 & - & 2.48 & $<0.001$ \\
\hline
\end{tabular}

\section{DISCUSSION}

In the present study, we compared the characteristics of patients with diabetes mellitus infected with COVID-19 who had unfavorable hospital admission course with those who has sound admission course and we analyzed the risk factors that predict the worse admission course for the patients. Our study showed that the independent risk factors associated with increased in-hospital mortality, ICU admission due to multiorgan failure or mechanical ventilation were the level of HBA1c, underlying hypertension, age more than 65 years, CT severity scoring, oxygen saturation less than 93, lymphopenia and high IL6, AST at time of admission.

In our study, the patients with the unfavorable course were significantly older, had higher BMI had many comorbidities like hypertension, hyperlipidemia, chronic pulmonary disease than the other group. Recent studies have demonstrated older age as a risk factor for mortality in patients infected with COVID-19 ${ }^{(\mathbf{3}, \mathbf{9}, \mathbf{1 0})}$. The age dependent defects in humeral and cellular immune function and the amplified inflammatory cytokines production may cause a poor immune response to viral replication (11). Likewise, the unfavorable prognosis of elderly patients with diabetes 
may be due to the association of many comorbidities, and the poor glycemic control.

Although fever and cough were common presenting symptoms in both groups, yet dyspnea tachypnea, tachycardia and low oxygen saturation were significant common presentations in the unfavorable course group. Our results showed that the presence of hypoxia at the time of admission was an independable predictive factor for the unfavorable course. Hypoxia at presentation should alert that the patient might benefit from early hospital admission, close monitoring, oxygen therapy to improve prognosis and decrease mortality.

Previous studies showed that DM increased the risk of mortality in patients infected by COVID-19 $(\mathbf{6 , 1 2 , 1 3 )}$. Moreover, the degree of DM control is an important predictor of the hospital admission course as showed by our study. Our results showed that a high HBA1c level is an independent risk factor for poor prognosis of admitted patients infected with COVID19. Guo et al. ${ }^{(6)}$ stated that hyperglycemia increases the risk of excessive inflammatory response and release of enzymes responsible for tissue damage.

In this study, we compared the laboratory characteristics of patients with favorable and unfavorable hospital admission course at admission. IL 6 was significantly higher in the unfavorable group. In quantifying the risk of poor clinical outcomes, we showed that high IL6 at the time of admission increases the odds of unfavorable hospital admission course significantly. This result support the concept of cytokine storm resulted from the release of inflammatory factors during COVID-19 infection plays a critical role in the progression to severe form of the disease $^{(14)}$.

In our study, lymphopenia and eosinopenia were more significantly reported in patients with unfavorable course group in contrast to neutropenia, which was more common in favorable course group. Multivariate analysis revealed that lymphopenia at presentation is an independent predictor of unfavorable admission course, this finding was in concordance with previous study stated that lymphopenia increases the risk of mortality in diabetic patients infected with COVID19 ${ }^{(\mathbf{1 0})}$. Severe form of infection with COVID19 is associated with the destruction of lymphocytes, cytokine storm and defect in cellular immune response ${ }^{(15)}$, which play roles in the evolution of acute respiratory distress syndrome.

Based on our finding, serum AST and ALT were significantly higher in the unfavorable course group, moreover, multivariate analysis revealed that high AST level at admission was an independent risk predictor for unfavorable hospital admission course, this may support the concept of infection of liver cells with COVID-19 ${ }^{(16)}$ and hypoxia associated liver cell injury in critically ill patients infected with COVID19 ${ }^{(17)}$.

Depending on chest radiographs, there were large numbers of false negative results due to lack of early abnormalities. Chest CT with thin-section is more sensitive than chest radiography, showing abnormal changes in the lung parenchyma in early stages of disease $^{(\mathbf{1 8 , 1 9 )}}$, so chest $\mathrm{CT}$ has become an important diagnostic tool during the pandemic of COVID-19 $9^{(20)}$.

In this study, we used a semi-quantitative scoring method using the amount of lung affection of the 3 right lobes and 2 left lobes to represent the degree of lung affection. We found that the CT-SS was significantly higher in cases with the unfavorable course when compared to cases with the favorable course; also the number of lobes affection is higher in cases with the unfavorable course when compared to cases with the favorable course. This is in agreement with Yang et al. (8), who reported that CT-SS with significantly higher in severe cases of COVID-19 than in mild cases.

Early recognition of patients at risk of poor prognosis during initial assessment helps to allocate the resources to improve the prognosis, monitoring process and mortality reduction of those at risk especially during COVID1-19 outbreak with limited resources.

To our knowledge, this is the first study to investigate the predictors either clinical laboratory or radiological for the course of hospital admission for patients with DM and COVID19, yet some limitation of the study related to the retrospective nature of the study and relatively small number of patients. Further multicenter studies with inclusion of a large number of patients should be performed in the future to clarify all predictors of the unfavorable course of COVID19 in different groups of patients.

\section{CONCLUSIONS}

Our findings suggest that significant predictors of unfavorable admission course in COVID19 patients with diabetes were old age, presence of hypertension, hypoxia at presentation, in addition to high HBA1c, AST, IL6, CT severity scoring and low lymphocyte.

Competing interests: The authors declare that they have no competing interests.

Funding: No funding was obtained for this study.

\section{REFERENCES}

1. Wang Y, Wang Y, Chen Y et al. (2020): Unique epidemiological and clinical features of the emerging 2019 novel coronavirus pneumonia (COVID-19) implicate special control measures. J Med Virol., 92(6):568-576.

2. Sun J, He W, Wang $L$ et al. (2020): COVID-19: Epidemiology, evolution, and cross-disciplinary perspectives. Trends Mol Med., 26(5):483-495.

3. Zhou F, Yu T, Du R et al. (2020): Clinical course and risk factors for mortality of adult inpatients with COVID-19 in Wuhan, China: a retrospective cohort study. Lancet, 395(10229):1054-1062.

4. Bertoni A, Saydah S, Brancati F (2001): Diabetes and the risk of infection-related mortality in the U.S. Diabetes Care, 24(6):1044-1049.

5. Kornum J, Thomsen R, Riis A et al. (2007): Type 2 diabetes and pneumonia outcomes: A population-based cohort study. Diabetes Care, 30(9):2251-2257. 
6. Guo W, Li M, Dong Y et al. (2020): Diabetes is a risk factor for the progression and prognosis of COVID-19. Diabetes Metab Res Rev., 36(7): 3319.

7. Chen T, Wu D, Chen H et al. (2020): Clinical characteristics of 113 deceased patients with coronavirus disease 2019: Retrospective study. https://www.bmj.com/content/368/bmj.m1091

8. Yang R, Li X, Liu H et al. (2020): Chest CT severity score: An imaging tool for assessing severe COVID-19. Radiol Cardiothorac Imaging, 2(2):47-55.

9. Wu C, Chen X, Cai Y et al. (2020): Risk factors associated with acute respiratory distress syndrome and death in patients with coronavirus disease 2019 pneumonia in Wuhan, China. JAMA Intern Med., 180(7):934-38.

10. Shi Q, Zhang X, Jiang F et al. (2020): Clinical characteristics and risk factors for mortality of COVID19 Patients with Diabetes in Wuhan, China: A TwoCenter, Retrospective Study. Diabetes Care, 43(7):1382-1391.

11. Opal S, Girard T, Ely E (2005): The immunopathogenesis of sepsis in elderly patients. In: Clinical Infectious Diseases. Clin Infect Dis., 41: 504512.

12. Roncon L, Zuin M, Rigatelli G et al. (2020): Diabetic patients with COVID-19 infection are at higher risk of ICU admission and poor short-term outcome. J Clin Virol., 127: 104354.

13. Fang L, Karakiulakis G, Roth $M$ (2020): Are patients with hypertension and diabetes mellitus at increased risk for COVID-19 infection? Lancet Respir Med., 8(4):21-28.

14. Liu F, Li L, Xu M et al. (2020): Prognostic value of interleukin-6, C-reactive protein, and procalcitonin in patients with COVID-19. J Clin Virol., 127: 104370-76.

15. Channappanavar R, Perlman S (2017): Pathogenic human coronavirus infections: causes and consequences of cytokine storm and immunopathology. Semin Immunopathol., 39(5):529-539.

16. Yeo C, Kaushal S, Yeo D (2020): Enteric involvement of coronaviruses: is faecal-oral transmission of SARSCoV-2 possible? Lancet Gastroenterol Hepatol., 5(4):335-337.

17. Zhang C, Shi L, Wang $\mathbf{F}$ (2020): Liver injury in COVID-19: management and challenges. Lancet Gastroenterol Hepatol., 5(5):428-430.

18. Paul N, Roberts H, Butany J et al. (2004): Radiologic pattern of disease in patients with severe acute respiratory syndrome: The Toronto experience. Radiographics, 24(2):553-563.

19. Ng M, Lee E, Yang J et al. (2020): Imaging profile of the COVID-19 infection: Radiologic findings and literature review. Radiol Cardiothorac Imaging., 2(1):200034.

20. Youmin G, Shiyuan L (2019): Radiological diagnosis of COVID-19: Expert recommendation from the Chinese Society of Radiology (1st Edition), Pp 1-12. http://isradiology.org/storage/app/media/CSR_Final\%2 0ver\%20of\%20Radiological\%20diagnosis\%20consensi s.pdf 